\title{
La zeolita y su efecto en la eficiencia del nitrógeno en arroz y maíz
}

\author{
Zeolite and its effect on nitrogen efficiency in rice and corn
}

\section{Miguel Soca1; Martha Constanza Daza T. ${ }^{2}$}

\footnotetext{
${ }^{1}$ Ph D. Dirección de Suelos, Ministerio de Agricultura, La Habana, Cuba, programas@minag.cu.

${ }^{2}$ Docente Asociada. Universidad del Valle, Colombia, martha.daza@correounivalle.edu.co.
}

Citar: SOCA, M.; CONSTANZA, M. 2015. La zeolita y su efecto en la eficiencia del nitrógeno en arroz y maíz. Rev. Cienc. Agr. 32(2):46 - 55.

\section{RESUMEN}

Este trabajo evaluó el efecto de la zeolita en las reducciones de volatilización del nitrógeno amoniacal (RVNA) y volumen de lixiviados (RVL) así como en rendimientos de arroz Oryza sativa L. y maíz Zea mays L. En el primer experimento bajo un Diseño Completamente al Azar (DCA) se evaluó el tamaño de partícula de zeolita (<1 mm, 1 - 2 mm, 2 - 3 mm y 3-5 mm) en RVNA. El segundo experimento, consistió en evaluar RVL con la aplicación de cuatro tratamientos: testigo (T), zeolita (Z), urea (U) y urea + zeolita (UZ). El tercer experimento se desarrolló en un cultivo de arroz, donde se evaluaron dos métodos de aplicación (al voleo y trasplante) con cuatro tratamientos (T, U, UZ10\% y UZ15\%) y su efecto en el rendimiento del cultivo. El cuarto experimento realizado en un campo de maíz con dos tratamientos (U y UZ15\%) evaluó el rendimiento del cultivo en dos suelos (aluvial y esquelético). Los tamaños de partícula entre 3 y $5 \mathrm{~mm}$ obtuvieron RVNA del 50\% y RVL del 48\%. La aplicación de zeolita en arroz obtuvo RVNA de 60\% y mejoró los rendimientos sin encontrar diferencias entre método de aplicación. También su aplicación mejoró el peso de grano por mazorca y el peso seco de grano de maíz. La zeolita mejoró la eficiencia de la fertilización nitrogenada.

Palabras clave: Volatilización, urea, lixiviación, clinoptilonita. 


\begin{abstract}
This study evaluated the effect of zeolites on ammonia volatilization reductions (AVR) and leaching volume (LV), as well as on rice Oryza sativa L. and maize Zea mays L. yields. The first experiment was conducted in a completely random design (CRD) and evaluated the influence of zeolite particle size ( $<1 \mathrm{~mm}, 1-2 \mathrm{~mm}, 2$ - $3 \mathrm{~mm}$, and 3-5 mm) on AVR. The second experiment evaluated VL in the application of four treatments: control (T), zeolite (Z), urea (U), and urea + zeolite (UZ). The third experiment was conducted in rice and evaluated the effect of two application methods (random spread and transplantation) and four treatments (T, U, UZ 10\% and UZ 15\%) on crop yield. The fourth experiment was conducted in a corn crop using two treatments ( $U$ and UZ15\%), there crop yield in two soils (alluvial and skeletal) was evaluated. Particle sizes between 3 and $5 \mathrm{~mm}$ showed an AVRof $50 \%$ and LV of $48 \%$. Zeolites application in rices howed an AVR of 60\% and increased yields with out differences between application methods. Its application also improved grain weight per ear and dry grain weight. Zeolites improved the efficiency of nitrogen fertilization.
\end{abstract}

Key words: Volatilization, urea, leaching, clinoptilolite.

\section{INTRODUCCIÓN}

La eficiencia en el uso del nitrógeno por los cultivos, varía entre 33 y 50\%, debido entre otros factores a la fluctuación temporal y espacial de los nitratos en el perfil del suelo (Hirel et al., 2011). Cuando se utiliza fertilizantes nitrogenados solo una parte del nitrógeno (menos del 50\%) es absorbido por las plantas (Colling et al., 2007), mientras que otra permanece en las capas más profundas del perfil y se lixivia (Hatch et al., 2002), provocando contaminación de acuíferos (Delgado et al., 2000). La lixiviación de nitratos se ve incrementada por la cantidad y la forma de aplicación de las láminas de riego ya sea de forma continua o intermitente (Tafteh y Reza, 2012). Otra fracción importante se pierde por volatilización en formas reducidas de nitrógeno $\left(\mathrm{NH}_{3}\right)$, donde el $67 \%$ de estas emisiones a la atmosfera desde el suelo y la agricultura son de carácter antrópico proveniente de fertilizantes nitrogenados principalmente (Fowler et al., 2013). En este sentido, es importante buscar alternativas para aumentar la eficiencia de la fertilización nitrogenada en la agricultura, como es el uso de zeolitas.
Las zeolitas, son aluminosilicatos, del grupo de los tectosilicatos cuya estructura tridimensional permite el intercambio iónico sin cambios en su estructura atómica (Osorio, 2014). Son de origen sedimentario o volcánico y poseen una alta capacidad de intercambio catiónico, favoreciendo la retención de iones $\mathrm{NH}_{4}^{+}$y otros cationes proveniente de los fertilizantes minerales (He et al., 2002).

La incorporación de zeolitas naturales en la formulación de fertilizantes minerales puede ser una alternativa para favorecer la retención del $\mathrm{NH}_{4}^{+}$y otros cationes provenientes de los fertilizantes (He et al., 2008). De acuerdo con De Campos et al. (2013) estas pueden actuar ya sea como abonos de liberación lenta incrementando el uso de nutrientes como el nitrógeno y el fósforo o como enmiendas al aumentar la capacidad de retención de humedad en los suelos. De igual manera, han sido reportados los beneficios de su aplicación en la reducción de la volatilización del nitrógeno hasta en un $47 \%$ cuando se combina con fertilizantes nitrogenados como la urea (Urquiaga y Zapata, 2002). Las formulaciones de zeolita con urea, han permitido reducir dosis de aplicación, 
sin disminución de los rendimientos, en cultivos como trigo (Osuna et al., 2012), Soja (Rodríguez y Gatti, 2010), hortalizas y papa (John et al., 2001), avena (Flórez et al., 2007), cebolla puerro (Álvarez et al., 2010), girasol (Gholamhoseini et al., 2013) y maíz (González et al., 2012).

Las zeolitas se han aplicado como mejorador de diferentes de suelos y cultivos, a voleo o de forma localizada, en el fondo del surco a ambos lados de la semilla y a una profundidad de $10 \mathrm{~cm}$, provocando en estos, un incremento de la Capacidad de Intercambio Catiónico y mayores contenidos en los suelos de fósforo y potasio (Febles et al., 2014).

El objetivo de éste trabajo, fue evaluar la aplicación de zeolita de origen cubano junto a la urea en los rendimientos de arroz Oryza sativa variedad INIAP-14 y en el cultivo del maíz Zea mays variedad US-536, así como en la volatilización del nitrógeno y en la pérdida de humedad por lixiviación.

\section{MATERIALES Y MÉTODOS}

Este experimento se llevó a cabo en las instalaciones del Instituto de Suelos de La Habana Cuba ubicado en las coordenadas $23^{\circ} 7^{\prime} \mathrm{LN}$ y $82^{\circ} 23^{\prime} \mathrm{LO}$, a una altura de $5 \mathrm{msnm}$. La temperatura y precipitación promedio anual es de $25,2^{\circ} \mathrm{C}$ y $1189 \mathrm{~mm}$ al año respectivamente. Los suelos utilizados pertenecen al orden taxonómico typic andoaquept caracterizados por sus colores grisáceos con drenaje interno deficiente. Se realizó un muestreo de suelos aleatoria de la parcela correspondiente a este tipo de suelo para minimizar la heterogeneidad siguiendo los lineamientos de Osorio y Casamitjana (2011).

La zeolita utilizada, provino del yacimiento de Tasajera compuesta por $85 \%$ de clinoptilonita y heulantita, $10 \%$ de modernita y el 5\% restante de otros minerales. El análisis mineralógico con difracción de Rayos X y la determinación de su composición química, se realizó con la colaboración del Centro de Investigaciones y Proyectos para la Industria Minero-Metalúrgica (CIPIMM) (Tabla 1). Esta zeolita es de tipo cálcico sódica con alta capacidad de intercambio catiónico y bajo contenido alumínico.

Efecto del tamaño de partícula de zeolita en la volatilización del nitrógeno. Los ensayos se llevaron a cabo en condiciones controladas en macetas de $5 \mathrm{~kg}$ de capacidad con suelo previamente tamizado por $2 \mathrm{~mm}$ y se evaluaron las pérdidas de nitrógeno amoniacales por volatilización presentadas de acuerdo con el tamaño de partícula después de transcurridos 45 días. Para esto, se aplicó una dosis equivalente de urea de $120 \mathrm{~kg}$ $\mathrm{hm}^{-2}$ más 15\% (con respecto al peso del suelo) de zeolita. Los tamaños de partícula evaluados fueron (<1mm, 1-2mm, 2-3mm y 3-5mm). Las pérdidas de nitrógeno por volatilización se determinaron siguiendo la metodología descrita por Lara y Trivelin (1997), la cual consistió en la instalación de espumas de poliuretano impregnadas con solución $0,5 \mathrm{~N}$ de $\mathrm{H}_{2} \mathrm{SO}_{4}$ y glicerina al $3 \%$ dentro de un colector tipo semiabierto estático, el cual absorbe la cantidad de amoniaco perdido desde el suelo y limita la entrada de nitrógeno desde la atmósfera.

Tabla 1. Análisis químico y composición catiónica de la zeolita utilizada en el presente estudio.

\begin{tabular}{rrrrrrrrrrrrr}
\hline $\mathrm{Si02}$ & $\mathrm{Al}_{2} \mathrm{O}_{3}$ & $\mathrm{MgO}$ & $\mathrm{CaO}$ & $\mathrm{N}_{2} \mathrm{O}$ & $\mathrm{K}_{2} \mathrm{O}$ & $\mathrm{F}_{2} \mathrm{O}_{3}$ & $\mathrm{PPI}^{*}$ & $\mathrm{CICT}$ & $\mathrm{Ca}^{+2}$ & $\mathrm{Mg}^{+2}$ & $\mathrm{~K}^{+}$ & $\mathrm{Na}^{+}$ \\
\hline \multicolumn{1}{c}{$\%$} & $\%$ & & & \multicolumn{7}{c}{$\mathrm{Cmol}^{(+)} \mathrm{kg}^{-1}$} & \\
\hline 66,0 & 10,1 & 0,4 & 2,9 & 2,9 & 0,8 & 1,8 & 15,0 & 138,0 & 92,0 & 4,0 & 9,0 & 34,0 \\
\hline
\end{tabular}

*PPI= Pérdidas por Ignición de gases volátiles; CICT= Capacidad de Intercambio Catiónico Total. 
Efecto de la zeolita en la lixiviación. Se establecieron macetas de $5 \mathrm{~kg}$ con suelo con el objetivo de evaluar la influencia de la aplicación de zeolita en la generación de lixiviados. Debajo de cada maceta se colocaron bolsas plásticas para recoger luego de transcurridas 24 horas, los lixiviados de cada tratamiento. La zeolita utilizada con tamaño de partículas de 1-3mm y la urea fueron secadas y mezcladas homogéneamente antes de ser ubicadas en la maceta. Los tratamientos considerados fueron: testigo, urea en dosis de $120 \mathrm{~kg} \mathrm{hm}^{-2}$, zeolita en dosis de $18 \mathrm{~kg} \mathrm{hm}^{-2}$ y la mezcla de urea más zeolita en las proporciones anteriores. Se determinó el volumen de los lixiviados recogidos al cabo de 45 días, mediante bolsas plásticas localizadas en la parte inferior de las macetas. Para cada tratamiento se dispuso de tres repeticiones para un total de 12 unidades experimentales.

\section{Efecto de la zeolita en la producción de masa seca en arroz Oryza sativa var.INIAP-14. Se rea-} lizó en diciembre de 2012, un ensayo en $1000 \mathrm{~m}^{2}$ en cultivos de arroz variedad INIAP-14en un diseño de Bloques Completos al Azar. Cada bloque tuvo un área de $200 \mathrm{~m}^{2}$ los cuales fueron separados $1 \mathrm{~m}$ entre ellos para evitar efectos de borde. Los tratamientos planteados en un área de $50 \mathrm{~m}^{2}$ cada uno fueron: testigo el cual no tuvo ninguna aplicación, urea en dosis de $120 \mathrm{~kg} \mathrm{hm}^{-2}$, Urea+zeolita (10\%), Urea+zeolita (15\%) con cinco repeticiones por tratamiento. Se empleó una densidad de siembra de 200 plantas $\mathrm{m}^{-2}$ para garantizar 350 - 400 panículas $\mathrm{m}^{-2}$. Se evaluaron dos métodos de siembra y aplicación de materiales: trasplante $\left(45 \mathrm{~kg} \mathrm{hm}^{-2}\right.$ de semilla)y al voleo(100 $\mathrm{kg} \mathrm{hm}^{-2}$ de semilla). En el método de trasplante, la aplicación de urea y/o zeolita se realizó en el sitio de siembra incorporándola a una profundidad de $0,02 \mathrm{~m}$ y en el método al voleo la aplicación de materiales se realizó esparciéndolos uniformemente e incorporándolos a 0,03m de profundidad con un rodillo compactador. Se evaluó los rendimientos del cultivo al cabo de su ciclo de producción por área de cada tratamiento $\left(50 \mathrm{~m}^{2}\right)$, teniendo en cuenta del total cosechado, el producido de arroz blanco listo para consumo.

\section{Efecto de la zeolita en el rendimiento de maíz} Zea mays L. variedad US-536. Se utilizaron dos tipos de suelos vertisoles: aluvial y esquelético. Los suelos aluviales utilizados se caracterizan por la ausencia de horizontes genéticos bien diferenciados y han sido formados por flujos hídricos lentos en su mayoría sobre depósitos carbonatados (Hernández et al., 2014). Los suelos esqueléticos se caracterizan por tener perfil tipo ACD o AD poco profundo $(<0,20 \mathrm{~m})$, con presencia significativa de grava y afectados por erosión moderada a severa al encontrarse en relieve montañoso. Se utilizó un Diseño Completo al Azar. En las parcelas experimentales de un $\mathrm{hm}^{2}$ se sembró maíz variedad INIAP-14. Se establecieron dos tratamientos: urea en dosis $120 \mathrm{~kg} \mathrm{hm}^{-2} \mathrm{y}$ urea $+15 \%$ de zeolita con cuatro repeticiones por tratamiento. Como variables de respuesta se contemplaron el peso de mazorca y rendimiento de grano, para lo cual se seleccionaron10 plantas al azar de cada unidad experimental, se pesaron en fresco y luego fueron desgranadas utilizando balanza electrónica; los granos obtenidos fueron secados en estufa por 24 horas a $50^{\circ} \mathrm{C}$.

Los datos obtenidos fueron analizados aplicando estadísticas descriptivas. Se verificó la distribución normal y la homogeneidad de los datos mediante las pruebas de ShapiroWilk y Lavene, respectivamente. Se realizó el Análisis de Varianza (ANDEVA) para determinar la existencia de diferencias significativas y se aplicó el test de Tukey $(p<0.05)$ para comparar medias de tratamiento. 


\section{RESULTADOS Y DISCUSIÓN}

\section{Efecto del tamaño de partícula de zeolita en} la volatilización del nitrógeno. La aplicación de zeolita con tamaño de partícula mayor a $1 \mathrm{~mm}$ redujo significativamente la volatilización del nitrógeno a los 45 días después de la aplicación de la urea (Tabla 2). La reducción de emisiones de nitrógeno en formas amoniacales en los tratamientos con zeolita estuvo entre 7,3\% y 56,9\% con respecto a la aplicación de la urea sin enmienda. A mayor tamaño de partícula, la reducción de la volatilización del nitrógeno fue menor, siendo las partículas entre 3 - $5 \mathrm{~mm}$ significativamente más efectivas que aquellas menores a $3 \mathrm{~mm}$. El mayor tamaño de partícula redujo la volatilización en 53,5, 44,8 y $42,9 \%$ con respecto a los tamaños $<1 \mathrm{~mm}$, $1-2 \mathrm{~mm}$ y $2-3 \mathrm{~mm}$. Similares resultados fueron hallados por Urquiaga y Zapata (2002) quienes reportaron reducción del orden de 46,6 y 47,8\% en la volatilización del nitrógeno al usar urea más 15\% de zeolita en suelos vertisoles pélicos y Grey nodulares, respectivamente. El mayor tamaño de partícula obtuvo el mejor comportamiento debido posiblemente a la similitud granulométrica entre la zeolita y el fertilizante utilizado, que garantizó mayor y mejor contacto entre partículas, favoreciendo la adsorción de amonio (Tarkalson, 2010; Malekian et al., 2011).
Estudios como el de Hernández, (2015) han mostrado que la mezcla urea + zeolita sobre el cultivo del arroz, han reducido hasta en un $50 \%$ las pérdidas del $\mathrm{N}$ por volatilización, con incrementos significativos en el rendimiento del cultivo. Según este investigador, la aplicación de urea sin enmienda generó pérdidas de nitrógeno por volatilización equivalentes al $77 \%$ más de lo generado por el suelo sin aplicación alguna.

Lazeolitajuntoconlaurea,redujosignificativamente las pérdidas de amoniaco, las cuales estuvieron por el orden de 61 (urea $+10 \%$ de zeolita) y $57 \%$ (urea $+15 \%$ de zeolita) en comparación con el testigo y del 42 y $46 \%$, respectivamente, en comparación con la aplicación de urea sola. Martins et al. (2015) cuantificaron en condiciones de campo las emisiones de $\mathrm{N}_{2} \mathrm{O}$ y volatilización de $\mathrm{NH}_{3}$ en cultivos de maíz en Oxisoles después de la fertilización con urea y zeolita y encontraron que en los diez primeros días las pérdidas de $\mathrm{N}$ fueron insignificantes pero entre 10 y 20 días las pérdidas aumentaron debido a procesos de nitrificación ocurridas, sin encontrar diferencias significativas entre tratamientos. De acuerdo a estos autores, el empleo de la zeolita no tuvo incidencia en la volatilización del $\mathrm{N}$ las cuales estuvieron por debajo del 4,5\%. No obstante, Zaman y Nguyen (2010) encontraron que la aplicación de zeolita

Tabla 2. Efecto del tamaño de partículas de la zeolita sobre las pérdidas por volatilización del $\mathrm{N}-\mathrm{NH}_{3}$ al ser utilizado como enmienda junto a fertilizante nitrogenado.

\begin{tabular}{llc}
\hline Tratamientos & mg-NH$_{3^{\cdot}} \mathbf{c m}^{-2} \mathbf{a ~ l o s ~} \mathbf{4 5}$ días \\
\hline Urea & & $371,0 \mathrm{a}$ \\
Urea+zeolita (15\%) & Menor 1mm & $344,0 \mathrm{a}$ \\
Urea+zeolita (15\%) & $1-2 \mathrm{~mm}$ & $290,0 \mathrm{~b}$ \\
Urea+zeolita (15\%) & $2-3 \mathrm{~mm}$ & $280,0 \mathrm{~b}$ \\
Urea+zeolita (15\%) & $3-5 \mathrm{~mm}$ & $160,0 \mathrm{c}$ \\
ESx & & 2,40 \\
\hline
\end{tabular}

Letras diferentes indican diferencias significativas (Tukey, $\alpha=0,05$ ) 
redujo la volatilización del $\mathrm{N}_{2} \mathrm{O}$ en $11 \%$ cuando se aplicaba junto a orina de vaca en parcelas de pastoreo vacuno compuesto por raygrass Lolium perenne L. y trébol blanco Trifolium repens L., debido principalmente a la adsorción de $\mathrm{NH}_{4}{ }^{+} \mathrm{y}$ por lo tanto ocurria en retraso en el proceso de nitrificación y la generación de $\mathrm{N}_{2} \mathrm{O}$.

\section{Efecto de la aplicación de zeolita en la lixivia-}

ción. La presencia de zeolita aumentó la capacidad de retención de humedad por lo que el volumen de lixiviados disminuyó sin embargo, no se encontraron diferencias significativas entre tratamientos debido a la alta dispersión de los datos registrados.

Similares resultados fueron hallados por Bigelow et al. (2004) quienes encontraron que a mayor cantidad de zeolita aplicada, la capacidad de retención de humedad del suelo se incrementaba significativamente, disminuyendo la lixiviación. La zeolita puede retener el $\mathrm{NH}_{4}^{+}$en sus partículas de manera interna y externa limitando la acción de las bacterias nitrificantes, permitiendo que el nitrógeno permanezca más tiempo en el suelo y por tanto disminuyendo su pérdida por lixiviación (Gilloway et al., 2003). Esto es par- ticularmente importante en suelos de textura arenosa donde debido a su alta conductividad hidráulica, las pérdidas de nitrógeno son mayores, por lo tanto la aplicación de zeolita permitiría la reducción potencial de los $\mathrm{NO}_{3}$ lixiviables favoreciendo el rendimiento del cultivo (Huang y Petrovic, 1994). Análogos efectos también fueron encontrados por Moradzadeh et al. (2014) quienes indican que con dosis de aplicación de zeolita potásica de hasta $8 \mathrm{~g} \mathrm{~kg}^{-1}$ de suelo, la lixiviación de nitratos, se redujo hasta en un 43\% con respecto al testigo sin aplicación de zeolita y atribuyen este resultado a la alta capacidad de retención de iones y agua que posee este tipo de minerales silicatados.

Resultados análogos fueron reportados por Gholamhoseini et al. (2013) quienes encontraron que la aplicación de urea junto a estiércol de vaca y $21 \%$ de zeolita redujo en aproximadamente $70 \%$ la lixiviación de nitratos en comparación con la urea sola en un cultivo de girasol; también encontraron que la aplicación de láminas de riego más bajas eran posibles cuando se utilizaba la zeolita junto al abono orgánico gracias al incremento en la capacidad de retención de humedad.



Figura 1. Efecto de la aplicación de la zeolita en combinación con urea en el volumen de lixiviados. 
Efecto de zeolita en la volatilización del nitrógeno y producción de masa seca en arroz Oryza sativa. En las pruebas de campo donde se establecieron parcelas de arroz con aplicación de zeolita, no se hallaron diferencias significativas en el rendimiento en comparación con la aplicación de la urea sola, pero si fueron halladas en comparación con el testigo (Tabla 3). La aplicación de $120 \mathrm{~kg}$ $\mathrm{hm}^{-2}$ de Urea +Zeolita aumento la adsorción del $\mathrm{NH}_{4}^{+}$en el suelo y logró retenerlo durante la etapa de máxima adsorción del arroz, favoreciendo el uso del nitrógeno (Tarkalson, 2010). El incremento del rendimiento en los tratamientos con zeolita, estuvo alrededor del $35 \%$ con respecto al testigo en los lotes donde se sembró el arroz por trasplante. Los rendimientos obtenidos en el sistema de siembra al voleo, fueron mayores alcanzando valores hasta de 6,5 $\mathrm{Mg} \mathrm{hm}^{-2}$ debido posiblemente, a que, con este método de siembra, se puede alcanzar mayor densidad de plantas. Nuevamente los tratamientos con zeolita estuvieron por encima de la urea y el testigo alcanzando incrementos del 5 y 38\% respectivamente. Similares resultados fueron reportados por Sepaskhah y Barzegar (2010) quienes alcanzaron incrementos de hasta 28 y $62 \%$ al utilizar $4 \mathrm{Mg} \mathrm{hm}^{-2}$ de zeolita y dosis de fertilizante nitrogenado de $80 \mathrm{~kg} \mathrm{hm}^{-2} \mathrm{con}$ respecto a la aplicación de urea sin zeolita y el testigo absoluto respectivamente.

Efecto de la zeolita en el rendimiento de maíz Zea mays cultivado en dos tipos de suelos. En el suelo aluvial, la aplicación de zeolita incrementó significativamente el peso de grano por mazorca y el peso seco de grano de maíz en 4,3 y 13,6\%, en su orden (Tabla 4). En el suelo esquelético, los rendimientos de grano alcanzados fueron mayores que en el suelo aluvial y estuvieron del orden de $4,42 \mathrm{~kg} \mathrm{hm}^{-2}$ con urea + zeolita y $4,16 \mathrm{~kg} \mathrm{hm}^{-2} \mathrm{con}$ la urea sola. Al igual que en el cultivo del arroz, la eficiencia en el uso del nitrógeno al igual que el acondicionamiento del medio edáfico pudieron ser las causas para obtener estos resultados.

La aplicación de zeolitas naturales en suelos ácidos produjeron mayores cosechas y mejor calidad de frutos de durazno albaricoque que la urea, al no permitir la reducción del $\mathrm{pH}$, el desbalance de bases, ni la liberación de elementos tóxicos como Mn y Al (Milosevic et al., 2013). Estos mismos autores, también encontraron que el uso de la zeolita sola no logra suministrar los nutrientes necesarios como N, y K para obtener altos rendimientos por lo que recomiendan usarla junto a abonos orgánicos e inorgánicos.

Tabla 3. Efecto de la aplicación de zeolita- en el cultivo del arroz variedad INIAP-14 en suelo Vertisoles y campos demostrativos.

\begin{tabular}{lcc}
\hline \multirow{2}{*}{ Tratamientos } & \multicolumn{2}{c}{ Mg hm $^{-2}$} \\
\cline { 2 - 3 } & $4,0 \mathrm{~b}$ & Siembra a voleo \\
\hline Testigo & $6,0 \mathrm{a}$ & $4,0 \mathrm{~b}$ \\
Urea $\left(120 \mathrm{~kg} \mathrm{hm}^{-2}\right)$ & $6,2 \mathrm{a}$ & $6,2 \mathrm{a}$ \\
Urea+zeolita $(10 \%)$ & $6,0 \mathrm{a}$ & $6,5 \mathrm{a}$ \\
Urea+zeolita $(15 \%)$ & $6,4 \mathrm{a}$ \\
\hline
\end{tabular}

Letras diferentes indican diferencias significativas (Tukey, $\alpha=0,05$ ) 
Tabla4. Influencia de la zeolita en la eficiencia del nitrógeno en el cultivo del maíz var. US-536.

\begin{tabular}{lcccc}
\hline \multirow{2}{*}{ Tratamientos } & \multicolumn{2}{c}{ Suelo Aluvial } & \multicolumn{2}{c}{ SueloEsquelético } \\
\cline { 2 - 5 } & $\begin{array}{c}\text { Peso grano/ } \\
\text { Mazorca (g) }\end{array}$ & $\begin{array}{c}\text { Grano seco } \\
\left(\mathbf{M g ~ h m}^{-2}\right)\end{array}$ & $\begin{array}{c}\text { Peso grano/ } \\
\text { Mazorca (g) }\end{array}$ & $\begin{array}{c}\text { Grano seco } \\
\left(\mathbf{M g ~ h m}^{-2}\right)\end{array}$ \\
\hline Urea & $171,68 \mathrm{~b}$ & $3,94 \mathrm{~b}$ & $191,78 \mathrm{a}$ & $4,16 \mathrm{a}$ \\
Urea+zeolita (15\%) & $179,43 \mathrm{a}$ & $4,56 \mathrm{a}$ & $199,20 \mathrm{a}$ & $4,42 \mathrm{~b}$ \\
ESx & 16,80 & 468,60 & 19,06 & 376,43 \\
\hline
\end{tabular}

a, b,c valores con letras iguales en una misma columna no difieren entre sí según la prueba de Tukey, $(\mathrm{p}<0.05)$

\section{CONCLUSIONES}

La aplicación de zeolita junto a la urea en tamaños de partícula entre $3-5 \mathrm{~mm}$, redujo hasta la volatilización del nitrógeno en forma amoniacal y el volumen de lixiviados con respecto a la aplicación de la urea sin ninguna enmienda.

La aplicación de zeolita en cultivos de arroz logró disminuir las pérdidas de nitrógeno por volatilización y aumentar los rendimientos con respecto al testigo y a la aplicación de urea sola. No se encontraron diferencias significativas entre formas de aplicación de estos materiales (trasplante 0 al voleo), en los rendimientos del arroz. El mayor rendimiento obtenido se logró cuando se realizó aplicación al voleo y aplicación de urea $+15 \%$ de zeolita. La aplicación de urea + $15 \%$ de zeolita aumento los rendimientos de maíz medidos en peso de grano por mazorca o en peso de grano seco.

\section{REFERENCIAS BIBLIOGRÁFICAS}

ÁLVAREZ, L., MÚNERA, V., NIVIA, D., RAMÍREZ, R. y GUTIÉRREZ, C. 2010. Efectos de la zeolita natural en la producción de cebolla puerro (Allíum porrum L.). Trabajo de Grado Ingeniero Agrónomo. Universidad Nacional de Colombia, Medellín. 60 p.

BIGELOW, C. BOWWMAN, D, y CASSEL, K. 2004. Physical properties of tree sand size classes amend with inorganic materials of Sphagnum peat moss for putting green rootzones. Crop Science. 44:900 - 907.
COLLING, H., DELGADO, A. y ALVA, K. 2007. Use of nitrogeno 15 isotopic techniques to estimate nitrogen cycling from a mustard cover crop to potatoes. Agronomy Journal. 99, 27 - 35.

DELGADO, J., FOLLET R. y SHAFFER, J. 2000. Simulation of nitrate nitrogen dynamic for cropping systems with different rooting depth. Soil Science Society American Journal. 64, 1050 - 1054.

DE CAMPOS BERNARDI, A. C., ANCHÃO OLIVIERA, P. P., DE MELO MONTE, M. B., y SOUZA-BARROS, F. 2013. Brazilian sedimentary zeolite use in agriculture. Microporous and Mesoporous Materials. 167, 16 - 21. http://doi.org/10.1016/j.micromeso. 2012.06.051.

FEBLES, J., BORSATTO, F. y SOCA, M. 2014. FercelClinoptilolite natural product to optimize the fertlization and reduce environmental pollution $\mathrm{p}$ 156-160. En: Memorias16 ${ }^{\text {th }}$ World Fertilizer Congress of CIEC. Technological Innovation for a Sustainable Tropical Agriculture. Río de Janeiro, Brasil.

FLOREZ, A., GALVIS, A., HERNÁNDEZ, T., DE LEÓN, F. y PAYÁN, F. 2007. Efecto de la adición de zeolita (clinoptilolita y mordenita) en un andosol sobre el ambiente químico edáfico y el rendimiento de avena. Interciencia. 32(10):692 - 696.

FOWLER, D., COYLE, M., SKIBA, U., SUTTON, M.A., NEIL CAPE, J., REIS, S., SHEPPARD, L.J., JENKINS, A. GRIZZETTI, B., GALLOWAY, J.N., VITOUSEK, P., LEACH, A., BOUWMAN, A.F., BUTTERBACH-Bah, K., DENTENER, F., STEVENSON, D. y AMANN, M. 2013. The global nitrogen cycle in the twenty first century. Philosophical Transactionsof TheRoyal Society B 368: 20130164. En: http://dx.doi.org/10.1098/rstb.2013.0164 and Maren Voss10; consulta: mayo, 2015. 
GHOLAMHOSEINI, M., GHALAVAND, M., KHODAEIJOGHAN, A., DOLATABADIAN, A., ZAKIKHANI, H. y FARMANBAR, E. 2013. Zeolite-amended cattle manure effects on sunflower yield, seed quality, water use efficiency and nutrient leaching. Soil Tillage Research. 126:193 - 202.

GILLOWAY, R., WEAVER, R. y MING, D. 2003. Nitrificacion in a zeoponic substrate. Plantand Soil. 256:341 - 378.

GONZÁLEZ, M., GÓMEZ, N., MUÑIZ, J., VALENCIA, F., GUTIÉRREZ, D. y FIGUEROA, H. 2012. Rendimiento del maíz de riego tratado con zeolita más fertilizante en el estado de Guerrero. Revista Mexicana de Ciencias Agrícolas. 3(6):1129 - 1144.

HATCH, D., GOULDING, K. y MURPHY, D. 2002. Agriculture: Potential source of water pollution, Nitrogen, pp 7-8. En: P. M. Haygarthy S. C. Jarvis (eds). Agriculture, hidrology, and Water Quality. Primera edición. Cromwell Press. Trowbridge UK. 493p.

HE, N., XE, M, y DING. Y. 2008. Computational study on IM-5 zeolite: What is its preferential location of $\mathrm{Al}$ and proton siting? Microporous and Mesoporous Materials.111(1-3):551 - 559.

HE, Z. L., CALVERT, D. V., ALVA, A. K., LI, Y. C., y BANKS, D. J. 2002. Clinoptilolite zeolite and cellulose amendments to reduce ammonia volatilization in a calcareous sandy soil. Plant and Soil. 247(2):253-260. http://doi. org/10.1023/A:1021584300322.

HERNÁNDEZ, A., LLANES, V., LÓPEZ, D. y RODRÍGUEZ, J. 2014. Características de vertisoles en áreas periféricas de La Habana. Cultivos Tropicales. 35(4):68 - 74.

HERNÁNDEZ, D. 2015. Mezcla de Urea y zeolita y su efecto sobre las pérdidas del nitrógeno y rendimiento del arroz. Agrobiologia, La Habana. 34 - 35.

HIREL, B., TÉTU, T., LEA, P. y DUBOIS, F. 2011. Improving nitrogen use efficiency in crops for sustainable agriculture. Sustainability. 3:1452 - 1485.

HUANG, Z. y PETROVIC, A.M. 1994. Clinoptilolite Zeolite Influence on Nitrate Leaching and Nitrogen Use Efficiency in Simulated Sand Based Golf Greens. Journal of Environmental Quality. 23:1190 - 1194.
JOHN, C., DEL VALLÍN, G., MORRERO, V., MUÑIZ, O., BELTRÁN, R., SOTLONGO, J., GIL, R., DELGADO, N. y CHONG, D. 2001. Generalización del empleo de la zeolita, como aditivo de la urea en cultivos de importancia económica, pp 193-195. En: Producción de cultivos en condiciones tropicales (ISBN: 959-711104-7). Primera edición. Instituto de Investigaciones Hortícolas. Quivican, Cuba, 280p.

LARA C. y TRIVELIN P.C. 1997. Eficiencia de un colector semi-abierto estático no cuantificado de N$\mathrm{NH}_{3}$ volatilizado da urea aplicado a un suelo. Revista brasileira de ciencia do solo. 14:481 - 487.

MALEKIAN, R., ABEDI-KOUPAI, J., y ESLAMIAN, S. S. 2011. Influences of clinoptilolite and surfactant-modifiedclinoptilolitezeolite on nitrate leaching and plant growth. Journal of Hazardous Materials. 185(2 - 3):970 - 976. http://doi.org/10.1016/j.jhazmat.2010.09.114

MARTINS, M.R., JANTALIA, C.P., POLIDORO, J.C. BATISTA, J.N., ALVES, B.J.R., BODDEY, R.M. y URQUUIAGA, S. 2015. Nitrous oxide and ammonia emissions from $\mathrm{N}$ fertilization of maize crop under no-till in a Cerrado soil. Soil Tillage Research. 151:75 - 81.

MILOSEVIC, T., MILOSEVIC, N. y GLISIC, I. 2013. Tree growth, yield, fruit quality attributes and leaf nutrient content of 'Roxana' apricot as influenced by natural zeolite, organic and inorganic fertilisers. Scientia Horticulturae. 156:131 - 139.

MORADZADEH, M., MOAZED, H., SAYYAD, G. y KHALEDIAN, M. 2014. Transport of nitrate and ammonium ions in a sandy loam soil treated with potassium zeolite - Evaluating equilibrium and non-equilibrium equations. Acta Ecológica Sínica. 34:342 - 350.

OSORIO, N.W. 2014. Manejo de nutrientes en suelos del trópico. Segunda edición. Vieco L. S.A.S., Medellín. 401 p.

OSORIO, W. y CASAMITJANA, M. 2011. Toma de muestras de suelo para evaluar la fertilidad del suelo. Suelos Ecuatoriales. 41(1):23 - 28.

OSUNA, E., RAMÍREZ, A., PAREDES, R., PADILLA, J. y BÁEZ, A. 2012. Eficiencia de la zeolita como aditivo de la urea e inoculación micorrízica en el cultivo de trigo. Revista Mexicana de Ciencias Agrícolas. 3(6):1101 1113. 
RODRÍGUEZ, M. B. y GATTI. J. P. 2010. Zeolitas como mejoradoras de la respuesta de la soja a los residuos de feedlot (CD). En: Memorias XXI Congreso Argentino de la Ciencia del suelo, Rosario. Potrero de los Funes, San Luis, Argentina.

SEPASKHAH, A. R. y BARZEGAR, M. 2010. Yield, water and nitrogen-use response of rice to zeolite and nitrogen fertilization in a semi-arid environment. Agricultural Water Management. 98(1):38 - 44. http:// doi.org/10.1016/j.agwat.2010.07.013.

TAFTEH, A. y REZA, A. 2012. Application of HYDRUS-1D model for simulating water and nitrate leaching from continuous and alternate furrow irrigated rapeseed and maize fields. Agricultural Water Management. 113:19 - 29.

TARKALSON, D. 2010. Clinoplolite zeolite influence on inorganic nitrogen in silt loam and sandy agricultural. Soil Science. 175:357 - 362.

URQUIAGA, S. y ZAPATA, F. 2002. Manejo eficiente de la fertilización nitrogenada de cultivos anuales en América Latina y el Caribe.EMBRAPA Porto Alegre. 52 $-53 \mathrm{p}$.

ZAMAN, M. y NGUYEN, M.L. 2010. Effect of lime or zeolite on N2O and N2 emissions from a pastoral soil treated with urine or nitrate- $\mathrm{N}$ fertilizer under field conditions. Agriculture. Ecosystems \& Environment. 136:254 - 261. 